Proyecciones Journal of Mathematics

Vol. 36, No 4, pp. 641-651, December 2017.

Universidad Católica del Norte

Antofagasta - Chile

\title{
On the solution of functional equations of Wilson's type on monoids
}

\author{
Iz-iddine EL-Fassi \\ Ibn Tofail University, Morocco \\ Abdellatif Chahbi \\ Ibn Tofail University, Morocco \\ and \\ Samir Kabbaj \\ Ibn Tofail University, Morocco \\ Received : December 2016. Accepted : September 2017
}

\begin{abstract}
Let $S$ be a monoid, $\mathbf{C}$ be the set of complex numbers, and let $\sigma, \tau \in \operatorname{Antihom}(S, S)$ satisfy $\tau \circ \tau=\sigma \circ \sigma=i d$. The aim of this paper is to describe the solution $f, g: S \rightarrow \mathbf{C}$ of the functional equation

$$
f(x \sigma(y))+f(\tau(y) x)=2 f(x) g(y), \quad x, y \in S,
$$

in terms of multiplicative and additive functions.

Subjclass [2010] : Primary 39B52.

Keywords : Wilson's functional equation, monoids, multiplicative function.
\end{abstract}




\section{Notation and terminology}

Throughout the paper we work in the following framework: A monoid is a semi-group $S$ with an identity element that we denote $e$, that is an element such that $e x=x e=x$ for all $x \in S$ (a semi-group is an algebraic structure consisting of a set together with an associative binary operation) and $\sigma, \tau: S \rightarrow S$ are two anti-homomorphisms (briefly $\sigma, \tau \in \operatorname{Antihom}(S, S)$ ) satisfying $\tau \circ \tau=\sigma \circ \sigma=i d$.

For any function $f: S \rightarrow \mathbf{C}$ we say that $f$ is $\sigma$-even (resp. $\tau$-even) if $f \circ \sigma=f$ (resp. $f \circ \tau=f$ ), also we use the notation $\check{f}(x)=f\left(x^{-1}\right)$ in the case $S$ is a group.

We say that a function $\chi: S \rightarrow \mathbf{C}$ is multiplicative, if $\chi(x y)=\chi(x) \chi(y)$ for all $x, y \in S$.

If $\chi: S \rightarrow \mathbf{C}$ is multiplicative and $\chi \neq 0$, then

$$
I_{\chi}:=\{x \in S \mid \chi(x)=0\} \text { is either empty or a proper subset of } S .
$$

If $S$ is a topological space, then we let $C(S)$ denote the algebra of continuous functions from $S$ into $\mathbf{C}$.

\section{Introduction}

Wilson's functional equation on a group $G$ is of the form

$$
f(x y)+f\left(x y^{-1}\right)=2 f(x) g(y), \quad x, y \in G,
$$

where $f, g: G \rightarrow \mathbf{C}$ are two unknown functions.

Special cases of Wilson's functional equation are d'Alembert's functional equation

$$
f(x y)+f\left(x y^{-1}\right)=2 f(x) f(y), \quad x, y \in G,
$$

and Jensen's functional equation

$$
f(x y)+f\left(x y^{-1}\right)=2 f(x), \quad x, y \in G .
$$

In [3] Ebanks and Stetkær studied the solutions $f, g: G \rightarrow \mathbf{C}$ of Wilson's functional equation (2.1) and the following variant of Wilson's functional (see [8])

$$
f(x y)+f\left(y^{-1} x\right)=2 f(x) g(y), \quad x, y \in G .
$$


They solve (2.3) and they obtained some new results about (2.1). We refer also to Wilson's first generalization of d'Alembert's functional equation

$$
f(x+y)+f(x-y)=2 f(x) g(y), \quad x, y \in \mathbf{R} .
$$

For more about the functional equation (2.4) see Aczél [1]. The solutions formulas of equation (2.4) for abelian groups are known.

In the same year Stetkær in [10] obtained the complex valued solution of the following variant of d'Alembert's functional equation

$$
f(x y)+f(\sigma(y) x)=2 f(x) f(y), \quad x, y \in \mathcal{S},
$$

where $\mathcal{S}$ is a semi-group and $\sigma$ is an involutive homomorphism of $\mathcal{S}$. The difference between d'Alembert's standard functional equation

$$
f(x y)+f(\tau(y) x)=2 f(x) f(y), \quad x, y \in \mathcal{S},
$$

and the variant (2.5) is that $\tau$ is an anti-homomorphism (on a group typically the group inversion). Some information, applications and numerous references concerning (2.5) and their further generalizations can be found e.g. in $[6,9,10]$.

Some general properties of the solutions of equation

$$
f(x y)+f(\sigma(y) x)=2 f(x) g(y)
$$

on a topological monoid $M$ equipped with a continuous involution $\sigma: M \rightarrow$ $M$ can be found in [9, Chapter 11].

Stetkær[11] proved a natural interesting relation between Wilson's functional equation (2.6) and d'Alembert's functional equation (2.2) and for $\sigma(x)=x^{-1}$. That is if $f \not \equiv 0$ is a solution of equation (2.6), then $g$ is a solution of equation (2.2). In [2] Chahbi et al. give a generalization of the symmetrized multiplicative Cauchy equation.

Recently, EL-Fassi et al. [5] obtained the solution of following functional equation

$$
f(x \sigma(y))+f(\tau(y) x)=2 f(x) f(y), \quad x, y \in \mathcal{S},
$$

where $\mathcal{S}$ is a semi-group and $\sigma, \tau$ are two anti-homomorphisms of $\mathcal{S}$ such that $\sigma \circ \sigma=\tau \circ \tau=i d$.

The main purpose of this paper is to solve the functional equation

$$
f(x \sigma(y))+f(\tau(y) x)=2 f(x) g(y), \quad x, y \in S
$$


where $S$ is a monoid and $\sigma, \tau \in \operatorname{Antihom}(S, S)$ such that $\sigma \circ \sigma=\tau \circ \tau=i d$. This equation is a natural generalization of (2.7) and of the following new functional equations

$$
\begin{gathered}
f(x \sigma(y))+f(\sigma(y) x)=2 f(x) g(y), \quad x, y \in S, \\
f(x \sigma(y))+f(\sigma(y) x)=2 f(x) f(y), \quad x, y \in S, \\
f(x \sigma(y))+f(\tau(y) x)=2 f(x), \quad x, y \in S, \\
f(x \sigma(y))+f(\sigma(y) x)=2 f(x), \quad x, y \in S,
\end{gathered}
$$

where $(S, \cdot)$ is a minoid and $\sigma, \tau \in \operatorname{Antihom}(S, S)$ such that $\sigma \circ \sigma=\tau \circ \tau=$ $i d$. Clearly, if $S$ is a group and $\check{f}=f$ with $\sigma(x)=x^{-1}$, then functional equation (2.10) becomes the symmetrized multiplicative Cauchy equation (see for instance [7] or [9, Theorem 3.21]). By elementary methods we find all solutions of (2.8) on monoid in terms of multiplicative functions. Finally, we note that the sine addition law on minoid given in $[4,9]$ is a key ingredient of the proof of our main result (Theorem 3.1).

\section{Solution of the functional equation (2.8)}

In this section we obtain the solution of the functional equation (2.8) on monoid. The following lemma will be used in the proof of Theorem 3.1.

Lemma 3.1. Let $S$ be a monoid and $\sigma \in \operatorname{Antihom}(S, S)$. Let $f, g: S \rightarrow \mathbf{C}$ be a solution of the functional equation

$$
f(x \sigma(y))=f(x) g(y), \quad x, y \in S .
$$

Then $g$ is a multiplicative function.

Proof. For all $x, y, z \in S$, we have

$$
\begin{aligned}
f(x) g(y z) & =f(x \sigma(y z))=f(x \sigma(z) \sigma(y)) \\
& =f(x \sigma(z)) g(y)=f(x) g(z) g(y),
\end{aligned}
$$

then $g(y z)=g(y) g(z)$ for all $x, z \in S$. This implies that $g$ is a multiplicative function. 
Theorem 3.1. Let $S$ be a monoid with identity element $e$, and $\sigma, \tau \in$ $\operatorname{Antihom}(S, S)$ such that $\sigma \circ \sigma=\tau \circ \tau=i d$ (where id denotes the identity map). The solutions $f, g: S \rightarrow \mathbf{C}$ of (2.8) are the following pairs of functions, where $\chi: S \rightarrow \mathbf{C}$ denotes a multiplicative function such that $\chi(e)=1$ :

(1) $f \equiv 0$ and $g$ arbitrary.

(2) $g=\frac{\chi+\chi \circ \sigma \circ \tau}{2}$ and $f=f(e) \chi \circ \sigma$, where $\chi \neq 0$ and $f(e) \in \mathbf{C} \backslash\{0\}$.

(3) If $\chi \neq \chi \circ \sigma \circ \tau$, then $g=\frac{\chi+\chi \circ \sigma \circ \tau}{2}$ and

(i) $f=f(e) \chi \circ \sigma$, where $f(e) \in \mathbf{C} \backslash\{0\}$,

or

(ii) $f=\alpha \chi \circ \sigma+(f(e)-\alpha) \chi \circ \tau$ for some constant $\alpha \in \mathbf{C} \backslash\{0, f(e)\}$, where $\chi \circ \sigma \circ \tau=\chi \circ \tau \circ \sigma$.

(4) If $\chi=\chi \circ \sigma \circ \tau$, and $S$ is generated by its squares, then

(i) $g(x)=\chi(x)$ and $f(x)=(A \circ \sigma(x)+f(e)) \chi \circ \sigma(x)$ for $x \in S \backslash I_{\chi}$,

(ii) $g(x)=f(x)=0$ for $x \in I_{\chi}$,

where $A: S \backslash I_{\chi} \rightarrow \mathbf{C}$ is a non-zero additive function such that $A \circ \sigma \circ$ $\tau=-A$.

Furthermore, if $S$ is a topological monoid, and $f, g \in C(S)$, then $\chi, \chi \circ \sigma, \chi \circ$ $\tau, \chi \circ \sigma \circ \tau \in C(S)$, and $A \circ \sigma \in C\left(S \backslash I_{\chi}\right)$.

Proof. It is elementary to check that the cases stated in the Theorem define solutions, so it is left to show that any solution $f, g: S \rightarrow \mathbf{C}$ of (2.8) falls into one of these case. We use in the proof similar Stetkaer's computations [10]. Let $x, y, z \in S$ be arbitrary. If we replace $x$ by $x \sigma(y)$ and $y$ by $z$ in (2.8), we get 


$$
f(x \sigma(z y))+f(\tau(z) x \sigma(y))=2 f(x \sigma(y)) g(z) .
$$

On the other hand if we replace $x$ by $\tau(z) x$ in (2.8), we infer that

$$
\begin{aligned}
f(\tau(z) x \sigma(y))+f(\tau(z y) x) & =2 f(\tau(z) x) g(y) \\
& =2 g(y)[2 f(x) g(z)-f(x \sigma(z))] .
\end{aligned}
$$

Replacing $y$ by $z y$ in (2.8), we obtain

$$
f(\tau(z y) x)=2 f(x) g(z y)-f(x \sigma(z y)) .
$$

It follows from (3.4) that (3.3) become

$f(\tau(z) x \sigma(y))+2 f(x) g(z y)-f(x \sigma(z y))=4 g(y) f(x) g(z)-2 g(y) f(x \sigma(z))$.

Subtracting this from (3.2) we get after some simplifications that

$f(x \sigma(z y))-f(x) g(z y)=g(y)[f(x \sigma(z))-f(x) g(z)]+g(z)[f(x \sigma(y))-f(x) g(y)]$.

With the notation

$$
f_{x}(y):=f(x \sigma(y))-f(x) g(y)
$$

equation (3.6) can be written as follows

$$
f_{a}(x y)=f_{a}(x) g(y)+f_{a}(y) g(x) .
$$

This shows that the pair $\left(f_{a}, g\right)$ satisfies the sine addition law for any $a \in S$. From the Known solution of the sine addition formula (see for example [4, Lemma 3.4]), we have the following possibilities.

If $f \equiv 0$ we deal with case $(\mathbf{1})$ in the Theorem. So during the rest of the proof we will assume that $f \not \equiv 0$. If we replace $(x, y)$ by $(e, \sigma(x))$ in (3.7), we get

$$
f(x)=f_{e} \circ \sigma(x)+f(e) g \circ \sigma(x), \quad x \in S .
$$

- Suppose that $f_{x}=0$ for all $x \in S$, then $f_{e}=0$, i.e., $f(x)=f(e) g \circ \sigma(x)$ for all $x \in S$, and hence $f(e) \neq 0$. Indeed, $f(e)=0$ would entail $f \equiv$ 0 , contradicting our assumption. From Lemma 3.1, we see that $g$ is a 
multiplication function. Substituting $f=f(e) g \circ \sigma$ into (2.8), we infer that $g=g \circ \sigma \circ \tau$. We may thus write $g=(g+g \circ \sigma \circ \tau) / 2$ which is the form claimed in the case (2).

- Now suppose that $f_{x} \neq 0$ for some $x \in S$.

If $f_{e} \neq 0$ then, from [4, Lemma 3.4], we see that there exist two multiplicative functions $\chi_{1}, \chi_{2}: S \rightarrow \mathbf{C}$ such that

$$
g=\frac{\chi_{1}+\chi_{2}}{2} .
$$

Case (3): If $\chi_{1} \neq \chi_{2}$, then $f_{e}=c\left(\chi_{1}-\chi_{2}\right)$ for some constant $c \in \mathbf{C} \backslash\{0\}$. From equality (3.9), we find after a reduction that

$$
f=\alpha \chi_{1} \circ \sigma+\beta \chi_{2} \circ \sigma
$$

where $\alpha=(2 c+f(e)) / 2$ and $\beta=f(e)-\alpha$. Substituting $f$ and $g$ in (2.8), we get after some simplification that

$$
\alpha \chi_{1} \circ \sigma(x)\left[\chi_{1} \circ \sigma \circ \tau(y)-\chi_{2}(y)\right]+\beta \chi_{2} \circ \sigma(x)\left[\chi_{2} \circ \sigma \circ \tau(y)-\chi_{1}(y)\right]=0
$$

for all $x, y \in S$. Since $\chi_{1} \neq \chi_{2}$ we get from the theory of multiplicative functions (see for instance [9, Theorem 3.18]) that both terms are 0 , so

$$
\left\{\begin{array}{l}
\alpha \chi_{1} \circ \sigma(x)\left[\chi_{1} \circ \sigma \circ \tau(y)-\chi_{2}(y)\right]=0 \\
\beta \chi_{2} \circ \sigma(x)\left[\chi_{2} \circ \sigma \circ \tau(y)-\chi_{1}(y)\right]=0
\end{array}\right.
$$

for all $x, y \in S$. Since $f \not \equiv 0$ at least one of $\alpha$ and $\beta$ is not zero.

Subcase (3.i): If $\alpha=0$ and $\beta \neq 0$, by (3.10) and $f \not \equiv 0$, for this to be the case we must have $\chi_{1} \neq 0, \chi_{2} \neq 0$ and $\chi_{2} \circ \sigma \circ \tau(y)=\chi_{1}(y)$ for all $y \in S$, then $f$ and $g$ have the desired form (3.i) with $\chi_{2}:=\chi$.

If $\alpha \neq 0$ and $\beta=0$, by (3.10) and $f \not \equiv 0$, for this to be the case we must have $\chi_{1} \neq 0, \chi_{2} \neq 0$ and $\chi_{1} \circ \sigma \circ \tau(y)=\chi_{2}(y)$ for all $y \in S$, then $f$ and $g$ have the desired form (3.i) with $\chi_{1}:=\chi$.

Subcase (3.ii): If $\alpha \neq 0$ and $\beta \neq 0$, by (3.10) and $f \not \equiv 0$, for this to be the case we must have $\chi_{1} \neq 0, \chi_{2} \neq 0, \chi_{1} \circ \sigma \circ \tau(y)=\chi_{2}(y)$ and $\chi_{2} \circ \sigma \circ \tau(y)=\chi_{1}(y)$ for all $y \in S$, with $\chi_{1}=\chi$, after some simplification, we obtain $\chi \circ \sigma \circ \tau=\chi \circ \tau \circ \sigma$ and the desired form (3.ii) of $f$ and $g$.

Case 4: If $\chi_{1}=\chi_{2}$ then letting $\chi:=\chi_{1}$ we have $g=\chi$. If $S$ is generated by its squares, then there exists an additive function $A: S \backslash I_{\chi} \rightarrow \mathbf{C}$ for which

$$
f_{e}(x)=\left\{\begin{array}{l}
A(x) \chi(x) \text { if } x \in S \backslash I_{\chi} \\
0 \text { if } x \in I_{\chi} .
\end{array}\right.
$$


Subcase (4.i): If $x \in S \backslash I_{\chi}$, then by (3.9) and (3.11), we get

$$
f(x)=(A \circ \sigma(x)+f(e)) \chi \circ(x)
$$

for all $x \in S \backslash I_{\chi}$. Substituting $f$ and $g$ in (2.8), we get after some simplification that

$(A \circ \sigma(x)+f(e))(\chi \circ \sigma \circ \tau(y)-\chi(y))+A \circ \sigma \circ \tau(y) \chi \circ \sigma \circ \tau(y)+A(y) \chi(y)=0$,

for all $x, y \in S \backslash I_{\chi}$. Suppose that $\chi \circ \sigma \circ \tau \neq \chi$. From (3.12) we infer that $A \equiv 0$, this contradicts with $f_{e} \neq 0$ on $S$. So $\chi \circ \sigma \circ \tau=\chi$ and $A \circ \sigma \circ \tau=-A$.

Subcase (4.ii): If $x \in I_{\chi}$, then $g(x)=f(x)=0$.

If $f_{e}=0$, then $f(x)=f(e) g \circ \sigma(x)$ for all $x \in S$, and hence $f(e) \neq 0$. Replacing $(x, y)$ by $(e, x)$ in $(2.8)$, we get

$$
f(\sigma(x))+f(\tau(x))=2 f(e) g(x), \quad x \in S .
$$

From (3.13) and $f(x)=f(e) g \circ \sigma(x)$ for all $x \in S$, we obtain $f(x)=$ $f(e) g \circ \tau(x)$ for all $x \in S$. So, $g$ is a solution of the functional equation

$$
g(x y)+g(\tau \circ \sigma(y) x)=2 g(x) g(y), \quad x, y \in S .
$$

Similar to the proofs of [10, Theorem 2.1], we find that $g=(\chi+\chi \circ \sigma \circ \tau) / 2$, where $\chi: S \rightarrow \mathbf{C}$ is multiplicative and $\chi \circ \sigma \circ \tau=\chi \circ \tau \circ \sigma$. Hence we are in case (2) or (3).

The continuity statement follows from [9, Theorem $3.18(\mathrm{~d})]$. This completes the proof of Theorem.

\section{Some consequences}

As immediate consequences of Theorem 3.1, we have the following corollaries.

Corollary 4.1. Let $S$ be a monoid with identity element $e$, and $\sigma, \tau \in$ $\operatorname{Antihom}(S, S)$ such that $\sigma \circ \sigma=\tau \circ \tau=i d$. The solutions $f, g: S \rightarrow \mathbf{C}$ of the functional equation

$$
f(x \sigma(y))+f(\sigma(y) x)=2 f(x) g(y), \quad x, y \in S
$$

are the following pairs of functions, where $\chi: S \rightarrow \mathbf{C}$ denotes a multiplicative function such that $\chi(e)=1$ : 
(1) $f \equiv 0$ and $g$ arbitrary.

(2) $g=\chi$ and $f=f(e) \chi \circ \sigma$, where $\chi \neq 0$ and $f(e) \in \mathbf{C} \backslash\{0\}$.

Furthermore, if $S$ is a topological monoid and $f, g \in C(S)$, then $\chi, \chi \circ \sigma \in$ $C(S)$.

Proof. It suffices to take $\tau(x)=\sigma(x)$ for all $x \in S$ in Theorem 3.1.

Corollary $4.2([5])$. Let $S$ be a monoid with identity element $e$, and $\sigma, \tau \in \operatorname{Antihom}(S, S)$ such that $\sigma \circ \sigma=\tau \circ \tau=i d$. The solutions $f: S \rightarrow \mathbf{C}$ of the functional equation

$$
f(x \sigma(y))+f(\tau(y) x)=2 f(x) f(y), \quad x, y \in S
$$

are the functions of the form $f=(\chi+\chi \circ \sigma \circ \tau) / 2$, where $\chi: S \rightarrow \mathbf{C}$ is a multiplicative such that:

(i) $\chi \circ \sigma \circ \tau=\chi \circ \tau \circ \sigma$, and

(ii) $\chi$ is $\sigma$-even or/and $\tau$-even.

Furthermore, if $S$ is a topological monoid and $f \in C(S)$, then $\chi, \chi \circ \sigma \circ \tau \in$ $C(S)$.

Proof. It suffices to take $g(x)=f(x)$ for all $x \in S$ in Theorem 3.1.

Corollary 4.3. Let $S$ be a monoid with identity element $e$, and $\sigma, \tau \in$ $\operatorname{Antihom}(S, S)$ such that $\sigma \circ \sigma=\tau \circ \tau=i d$. The solutions $f: S \rightarrow \mathbf{C}$ of the functional equation

$$
f(x \sigma(y))+f(\tau(y) x)=2 f(x), \quad x, y \in S
$$

are the functions of the form:

(1) $f \equiv 0$.

(2) $f=f(e)$, where $f(e) \in \mathbf{C} \backslash\{0\}$.

(3) If $S$ is generated by its squares, then

(i) $f(x)=A \circ \sigma(x)+f(e)$ for $x \in S \backslash I_{\chi}$,

(ii) $f(x)=0$ for $x \in I_{\chi}$,

where $A: S \backslash I_{\chi} \rightarrow \mathbf{C}$ is an additive function such that $A \circ \sigma \circ \tau=-A \neq 0$. Furthermore, if $S$ is a topological monoid, and $f \in C(S)$, then $A \circ \sigma \in$ $C\left(S \backslash I_{\chi}\right)$. 
Proof. It suffices to take $g(x)=1$ for all $x \in S$ in Theorem 3.1.

Corollary 4.4. Let $S$ be a monoid with identity element $e$, and $\sigma \in$ $\operatorname{Antihom}(S, S)$ such that $\sigma \circ \sigma=i d$. The solutions $f: S \rightarrow \mathbf{C}$ of the functional equation

$$
f(x \sigma(y))+f(\sigma(y) x)=2 f(x) f(y), \quad x, y \in S
$$

are the functions of the form $f=\chi$, where $\chi: S \rightarrow \mathbf{C}$ is a multiplicative such that $\chi$ is $\sigma$-even.

Proof. It suffices to take $g(x)=f(x)$ and $\tau(x)=\sigma(x)$ for all $x \in S$ in Theorem 3.1.

\section{Acknowledgments}

The author would like to express his most sincere gratitude to the two reviewers for a number of valuable comments which have led to essential improvement of the paper.

\section{References}

[1] J. Aczél and J. Dhombres, Functional equations in several variables, Cambridge University Press, New York, (1989).

[2] A. Chahbi, B. Fadli and S. Kabbaj, A generalization of the symmetrized multiplicative Cauchy equation, ActaMath. Hungar., 149, pp. 1-7, (2016).

[3] B. R. Ebanks and H. Stetkær, On Wilson's functional equations, Aequat. Math., 89, pp. 339-354, (2015).

[4] B. R. Ebanks and H. Stetkær, d'Alembert's other functional equation on monoids with an involution, Aequationes Math. 89, pp. 187-206, (2015).

[5] Iz. EL-Fassi, A. Chahbi and S. Kabbaj, The Solution of a class functional equations on semi-groups, Filomat, to appear. 
[6] PL. Kannappan, Functional equations and inequalities with applications, Springer, New York, (2009).

[7] H. Stetkær, On multiplicative maps, semi-group Forum, 63 (3), pp. 466-468, (2001).

[8] H. Stetkær, On a variant of Wilson's functional equation on groups, Aequat. Math., 68, pp. 160-176, (2004).

[9] H. Stetkær, Functional equations on groups, World Scientific Publishing Co, Singapore, (2013).

[10] H. Stetkær, A variant of d'Alembert's functional equation, Aequationes Math. 89, pp. 657-662, (2015).

[11] H. Stetkær, A link between Wilson's and dAlembert's functional equations, Aequat. Math. 90, pp. 407-409, (2015).

\title{
Iz-iddine El-Fassi
}

Department of Mathematics, Faculty of Sciences, Ibn Tofail University, BP 133, Kenitra, Morocco e-mail : izidd-math@hotmail.fr

\author{
Abdellatif Chahbi \\ Department of Mathematics, \\ Faculty of Sciences, \\ Ibn Tofail University, \\ BP 133, Kenitra, \\ Morocco \\ e-mail : abdellatifchahbi@gmail.com \\ and
}

\section{Samir Kabbaj}

Department of Mathematics, Faculty of Sciences, Ibn Tofail University, BP 133, Kenitra, Morocco e-mail : samkabbaj@yahoo.fr 